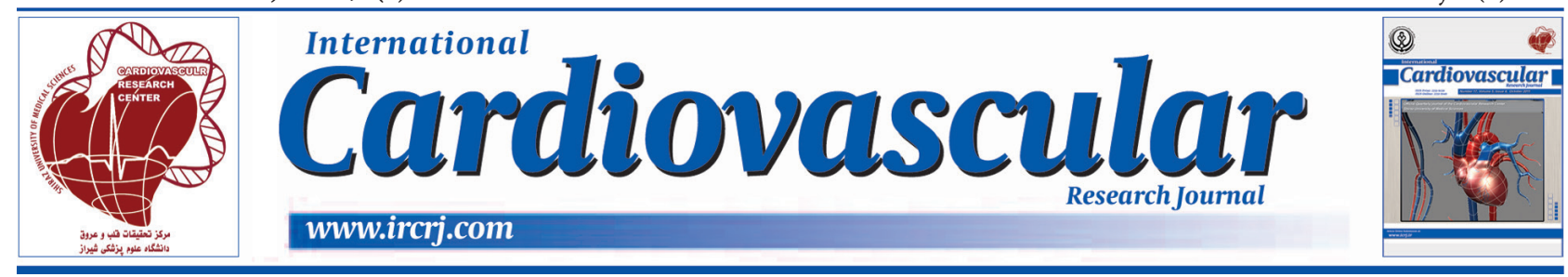

\title{
A Study on Hs-CRP, Carotid Intima Media Thickness, and Lipid Profile in Children of Patients with Premature CAD
}

\author{
Usha Dudeja Bindal,,${ }^{1 *}$ Santosh Kumar Gupta, ${ }^{2}$ Vivek Bindal, ${ }^{3}$ Mridul Kumar Daga, ${ }^{4}$ and Gaurav \\ Pradhan $^{5}$
}

${ }^{1}$ Department of Biochemistry, Dr. BS Ambedkar Medical College, New Delhi, India

${ }^{2}$ Department of Biochemistry, Maulana Azad Medical College, New Delhi, India

${ }^{3}$ Ganga Ram Institute of Post Graduate Medical Education and Research, New Delhi, India

${ }^{4}$ Department of Medicine, Maulana Azad Medical College, New Delhi, India

${ }^{5}$ Department of of Radiodiagnosis, Maulana Azad Medical College, New Delhi, India

A R T I C L E I N F O

Article Type:

Research Article

\section{Article History:}

Received: 15 Aug 2014

Revised: 31 May 2015

Accepted: 14 Jun 2015

\section{Keywords}

Atherogenesis

Cystatin-C

Carotid Intima-Media Thickness

\begin{abstract}
A B S T R A C T
Background: This study aimed to evaluate the risk markers of Coronary Artery Disease (CAD), so that appropriate lifestyle changes can be instituted early to prevent or delay development of the disease.

Objectives: This study aimed to evaluate the risk markers of CAD based on inflammation and arterial morphology; i.e., high sensitivity C-Reactive Protein (hs-CRP) and carotid artery Intima Media Thickness (IMT), in children of patients with premature CAD.

Patients and Methods: This was a case-control study with predetermined end points. It was conducted on 40 subjects randomly selected from the children of premature CAD patients and 40 age- and sex-matched healthy controls. Blood levels of hs-CRP, lipid profile, and carotid IMT were assessed for all the subjects. The relationship among these parameters was evaluated by univariate and multivariate analyses using the SPSS statistical software, version 22.0 and $\mathrm{P}<0.05$ was considered as statistically significant. Results: The mean values of Total Cholesterol (TC, $139.8 \pm 24$ vs. $111.3 \pm 14.7, \mathrm{P}<0.05$ ), Triglycerides (TG, $81.8 \pm 15.3$ vs. $63.4 \pm 10.8, \mathrm{P}<0.05$ ), Low Density Lipoprotein (LDL, $85.2 \pm 22.2$ vs. $57.5 \pm 14.1, \mathrm{P}<0.05)$, hs-CRP $(1.2 \pm 0.77$ vs. $0.79 \pm 0.27, \mathrm{P}<0.05)$ and carotid artery IMT $(0.49 \pm 0.09$ vs. $0.422 \pm 0.03, \mathrm{P}<0.05)$ were significantly higher in the cases compared to the controls. Also, both LDL/High Density Lipoprotein (HDL) and TC/HDL ratios were significantly higher in the cases compared to the controls. Moreover, a significant positive correlation was found between carotid IMT and hs-CRP. Conclusions: Children of patients with premature CAD showed a significantly higher incidence of dyslipidemia as well as significantly increased levels of the inflammation marker, hs- CRP, compared to the age- and sex-matched controls. This indicates that atherosclerosis is not only a disease of lipid deposition, but also an inflammatory process. This was further confirmed by demonstration of a significantly raised IMT.
\end{abstract}

Implication for health policy/practice/research/medical education:

This study evaluated non-conventional risk factors in children of patients with premature CAD. This is a relatively untouched field with a very scanty literature on risk assessment parameters. This pilot study showed significantly higher values of hs-CRP and carotid intima media thickness in this population. Thus, these parameters can potentially be used for future risk assessment of CAD and initiating preventive measures early in life.

\section{Background}

Coronary Artery Disease (CAD) is a major global health problem with high morbidity and mortality in the U.S.,

*Corresponding author: Usha Dudeja Bindal, C-302, Paradise Apartments, I.P. Extension, New Delhi 110060, India, Tel: +91-9868302541,

E-mail: ushadudeja@yahoo.com
Europe, and Southeast Asia (1). The prevalence of CAD is very high in both migrant Asian Indians and among the people of the Indian subcontinent (2). Furthermore, CAD affects Indians 5 - 10 years earlier compared to other communities (3) and its prevalence is 5 - 10 folds higher in patients under the age of 40 years (4). In spite of the lower 
rates of traditional risk factors in Indians (5), the prevalence rate of CAD in India is about $10 \%$, with a 2 -fold rise in urban population. An important feature of CAD in India is its prematurity and severity.

High prevalence rate of CAD in Indians is not fully explained by well documented traditional risk factors of CAD, such as age, male sex, family history of Myocardial Infarction (MI) and sudden death, dyslipidemia, smoking, hyperhomocysteinemia, high blood pressure, diabetes mellitus, obesity, sedentary lifestyle, stress, and inflammation $(6,7)$. Hence, a genetic component is believed to be involved (8-10). This familial predisposition aggravates the effects of conventional risk factors leading to higher CAD rates $(11,12)$.

The fact that atherosclerotic lesion is not simply a disease of lipid deposition but represents an aggregate of an inflammatory disease has been demonstrated by laboratory and clinical evidence (13). Fatty streak is the earliest type of lesion, which is common in infants and young children (14).

Highly sensitive C-Reactive Protein (hs-CRP) has also been found to be a good predictor of future coronary event in healthy men and women as well as in those with pre-existing cardiac disease (15). However, few studies have assessed hs-CRP in children with family history of premature CAD.

The early onset in the fetal arteries and silent progression of atherosclerosis is well known $(16,17)$. Measurement of carotid artery Intima Media Thickness (IMT) by noninvasive methods, such as ultrasound and color Doppler technique, is considered a reliable surrogate marker for preclinical atherosclerosis in high-risk children. Yet, paucity of data linking carotid IMT to CRP levels in children of premature CAD patients justifies further studies.

\section{Objectives}

Considering what was mentioned above, the present study was planned to evaluate the risk markers of CAD based on inflammation and arterial morphology; i.e., hs-CRP and carotid artery IMT, in children of patients with premature CAD in Indian population. In this way, appropriate lifestyle changes can be instituted early to prevent or delay development of CAD in young Indian individuals.

\section{Patients and Methods}

The present study was carried out in the department of Biochemistry, Medicine, and Radio diagnosis of Maulana Azad Medical College and the affiliated hospitals after gaining the approval of the institutional Ethics Committee. A total of 80 subjects, mostly children, were included in this study. They were divided into a study group (Group $\mathrm{I}, \mathrm{N}=40$ ) and a control group (Group II, $\mathrm{N}=40$ ). Written informed consents were obtained from all the subjects or their parents.

\subsection{Methodology}

This was a case-control study with predetermined end points.

\subsection{Selection of Cases and Controls}

Study group: This group included 40 subjects randomly selected from the children of patients with premature CAD who were admitted to the medical emergency and Coronary Care Unit (CCU) of Lok Nayak hospital. At first, a detailed history was taken from and physical clinical examination was performed for all the study subjects and their mothers or fathers admitted or treated for premature CAD. Details of the relevant laboratory investigations were also noted.

\subsection{Control Group}

For this group, 40 age- and sex-matched controls were chosen from the general population. The parents of these children had no history of CAD as evidenced by a detailed history, physical examination, and routine laboratory investigations.

\subsection{Inclusion Criteria}

The inclusion criterion of the study for the case group was suffering from proven premature CAD (MI, angina, and unstable angina or sudden death before the age of 45 years in fathers and 55 years in mothers). Also, the inclusion criteria for the control group were being age- and sex-matched with the cases and having no manifestation of CAD. On the other hand, the exclusion criteria of the study were having chronic liver disease and thyroid disorders, being on glucocorticoid therapy, and suffering from acute/chronic infections since these factors are known to affect the parameters under our investigation.

\subsection{Collection of Blood Samples}

After an overnight fasting, $5 \mathrm{ml}$ blood samples were taken from antecubital vein in all the subjects under aseptic precautions. In order to separate the sera collected in clean appendorfs, the blood samples were centrifuged at 2000 rpm for 10 minutes and were stored at $-70^{\circ} \mathrm{C}$ for estimation of hs-CRP and lipid profile in batch analysis.

\subsection{Estimation of $h s-C R P$}

Hs-CRP was analyzed by immunoassays based on ELISA method using commercially available kits. In fact, estimation of hs-CRP was based on the principle of a solid phase sandwich ELISA.

\subsection{Lipid Profile Estimation}

Lipid profile (TC, TG, HDL cholesterol, and LDL cholesterol) was analyzed through enzymatic methods using 'Autozyme's cholesterol enzymatic kit' by an autoanalyzer (Olympus AU 400®).

\subsection{Measurement of Carotid Artery Intima Media Thickness}

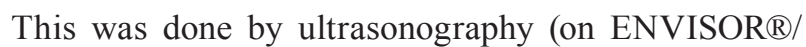
PHILIPS-HD 11®/ATL® USG machines) and/or color Doppler in the radiology department.

In each examination, the sonographer used different scanning angles (anterior and lateroposterior) to identify the greatest IMT, defined as the distance between the junction of the lumen and intima and that of the media and adventitia.18 Overall, three measurements of IMT were made in the right and left carotid arteries and were averaged to determine the mean IMT for each side and for both sides. 


\subsection{Statistical Analysis}

The results of parametric and non-parametric data were expressed as mean \pm Standard Deviation (SD) and median (range), respectively. Comparisons between the two groups were carried out using Fisher's exact test for discrete variables and Student's t-test for continuous variables. Additionally, univariate and multivariate analyses were performed to find out the role of independent risk factors considering the confounding factors. All the statistical analyses were performed using IBM $®$ SPSS $₫$ Statistical software, version 22.0. Confidence intervals were set at $95 \%$ and two-sided $\mathrm{P}$ value $\leq 0.05$ was considered as statistically significant.

\section{Results}

4.1 Demographic Features and Conventional Risk Factors

The subjects' age distribution along with the conventional risk factors of CAD, namely Body Mass Index (BMI), Systolic Blood Pressure (SBP), Diastolic Blood Pressure (DBP), and blood sugar, have been presented in Table 1. The results showed that among the cases, 25 children's fathers and 15 ones' mothers had premature CAD. Besides, the means of SBP, DBP, and BMI were significantly higher in the cases compared to the controls.

\subsection{Lipid Parameters}

The results indicated that the mean values of $\mathrm{TC}, \mathrm{TG}$, and LDL were significantly higher in the cases compared to the controls, whereas the mean of HDL was higher in the controls compared to the cases (Table 2). Both LDL/ HDL and TC/HDL ratios were also significantly higher in the cases in comparison to the controls. However, no significant difference was found between male and female children regarding the lipid parameters.

\subsection{Comparison of hs-CRP and Carotid IMT}

The results revealed a significant increase in hs-CRP and carotid artery IMT in the cases compared to the controls (Table 3). Besides, in comparison to female cases, male ones had significantly higher values of hs-CRP $(1.6 \pm 0.8$ vs. $1.0 \pm 0.6, \mathrm{P}=0.022)$ and Carotid IMT $(0.5 \pm 0.1$ vs. 0.4 $\pm 0.1, \mathrm{P}=0.008)$. However, no significant difference was observed between male and female controls regarding these parameters.

The correlations between hs-CRP and carotid IMT and lipid profile, blood pressure, and blood sugar were also studied (Table 4) and the results indicated a highly significant positive correlation between carotid IMT and hs-CRP.

In unconditional logistic regression multivariate analysis, the only parameters that retained statistical significance were blood sugar $(\mathrm{P}=0.025)$, TC $(\mathrm{P}=0.044)$, and DBP $(\mathrm{P}=$ 0.025). Although IMT did not reach statistical significance, its $\mathrm{P}$ value was 0.091 which shows that it may attain significance in larger series.

\section{Discussion}

Scanty data is available from limited studies on the relationship between hs-CRP and carotid artery IMT in children of patients with premature CAD. This implies that changes in serum hs-CRP levels and IMT in children of premature CAD patients in India have mostly remained unknown. The present study unraveled the importance of these parameters in children of patients suffering from premature CAD. This study was conducted in children and adolescents because of the well-known fact that atherogenic process begins in early childhood when fatty streaks develop in the intima of arteries. Strong and McGill studied aortic and coronary lesions in 4737 young subjects (10 -

\begin{tabular}{llll}
\hline \multicolumn{3}{l}{ Table 1. Demographics and Conventional Risk Factors in the Cases and Controls (Correlation is Significant at P $\leq 0.05)$} \\
\hline & Cases $($ Mean \pm SD) & Controls $($ Mean \pm SD) & P value \\
\hline Age (years) & $13.3 \pm 5.1$ & $12.2 \pm 4.1$ & $>0.05$ \\
BMI $\left(\mathbf{K g} / \mathbf{m}^{2}\right)$ & $18.7 \pm 3.4$ & $17.2 \pm 2.9$ & 0.042 \\
SBP $(\mathbf{m m ~ H g})$ & $109.6 \pm 14.2$ & $104.5 \pm 8.2$ & 0.05 \\
DBP $(\mathbf{m m ~ H g )}$ & $65.6 \pm 10.1$ & $58.8 \pm 6.5$ & 0.001 \\
Blood sugar $(\mathbf{m g} \%)$ & $76.4 \pm 7.1$ & $71.4 \pm 9.3$ & 0.009 \\
\hline
\end{tabular}

Abbreviations: BMI, body mass index; SBP, systolic blood pressure; DBP, diastolic blood pressure

\begin{tabular}{llll}
\hline \multicolumn{4}{l}{ Table 2. Lipid Parameters in the Cases and Controls $(\mathrm{P} \leq 0.05$ Denotes Statistical Significance) } \\
\hline Parameters & Cases $($ Mean \pm SD) & Controls $($ Mean \pm SD $)$ & P value \\
\hline TC $(\mathbf{m g} / \mathbf{d L})$ & $139.8 \pm 24$ & $111.3 \pm 14.7$ & $\leq 0.05$ \\
TG $(\mathbf{m g} / \mathbf{d L})$ & $81.8 \pm 15.3$ & $63.4 \pm 10.8$ & $\leq 0.05$ \\
HDL $(\mathbf{m g} / \mathbf{d L})$ & $37.8 \pm 5.2$ & $41.4 \pm 4.5$ & $\leq 0.05$ \\
LDL $(\mathbf{m g} / \mathbf{d L})$ & $85.2 \pm 22.2$ & $57.5 \pm 14.1$ & $\leq 0.05$ \\
LDL/HDL & $2.3 \pm 0.7$ & $1.4 \pm 0.4$ & $\leq 0.05$ \\
TC/HDL & $3.8 \pm 0.8$ & $2.7 \pm 0.5$ & $\leq 0.05$ \\
\hline
\end{tabular}

Abbreviations: TC, total cholesterol; TG, triglycerides; HDL, high density lipoprotein; LDL, low density lipoprotein

\begin{tabular}{llll}
\hline Table 3. Comparison of hs-CRP and Carotid IMT between the Cases and Controls & \\
\hline Parameters & Cases (Mean \pm SD) & Controls $($ Mean \pm SD) & \\
\hline High sensitivity C-reactive protein $(\mathbf{m g} / \mathbf{L})$ & $1.2 \pm 0.77$ & $0.79 \pm 0.27$ & $\leq 0.05$ \\
Carotid intima media thickness $(\mathbf{m m})$ & $0.49 \pm 0.09$ & $0.42 \pm 0.03$ & $\leq 0.05$ \\
\hline
\end{tabular}


Table 4. The Correlations between hs-CRP and Carotid IMT and Lipid Profile, Blood Pressure, and Blood Sugar (P $<0.05$ Denotes a Significant Positive Correlation)

\begin{tabular}{llll}
\hline & & hs-CRP & Carotid IMT \\
\hline SBP & Correlation Coefficient & 0.385 & 0.477 \\
DBP & P value & 0.014 & 0.002 \\
& Correlation coefficient. & 0.141 & 0.314 \\
TC & P value & 0.387 & 0.049 \\
& Correlation coefficient & 0.523 & 0.507 \\
TG & P value & 0.001 & 0.001 \\
& Correlation coefficient & 0.389 & 0.388 \\
HDL & P value & 0.013 & 0.013 \\
& Correlation coefficient & 0.086 & 0.110 \\
LDL & P value & 0.498 \\
& Correlation coefficient & 0.597 & 0.435 \\
LDL/HDL & P value & 0.463 & 0.005 \\
& Correlation coefficient & 0.003 & 0.279 \\
TC/HDL & P value & 0.325 & 0.081 \\
& Correlation coefficient & 0.041 & 0.309 \\
Blood sugar & P value & 0.347 & 0.053 \\
& Correlation coefficient & 0.028 & -0.083 \\
hs-CRP & P value & -0.103 & 0.610 \\
& Correlation coefficient & 0.526 & 0.810 \\
\hline
\end{tabular}

Abbreviations: BMI, Body Mass Index; SBP, Systolic Blood Pressure; DBP, Diastolic Blood Pressure; TC, Total Cholesterol; TG, Triglycerides; HDL, High Density Lipoprotein; LDL, Low Density Lipoprotein; hs-CRP, High Sensitivity C Reactive Protein; IMT, Intima Media Thickness

39 years of age) included in International Atherosclerosis Project, an autopsy study from 14 different countries (18). They showed that by 10 years of age, the autopsied aorta had fatty streak in the majority of subjects and some had coronary fatty streaks. Hence, it is justified to detect and track early dyslipidemia and other early markers of CAD from childhood.

Similarly, Sniderman et al. reported that children of patients with premature MI had significantly high lipid levels, except for HDL (19). Kelishadi et al. also demonstrated high levels of TC, TG, and LDL and low levels of HDL in children of patients with premature CAD (20). In the same line, Gulati et al. revealed that TC, LDL, and TG levels were significantly higher among children of patients with premature CAD compared to age- and sexmatched controls (21). In contrast, Khalil et al. indicated no significant difference between the children of patients with proven CAD and healthy parents concerning the lipid profile (22).

In our study, LDL-C/HDL-C and TC/HDL-C ratios were significantly higher in the cases compared to the controls. Widhalm et al. also reported a significant difference between at risk and non-risk groups of children regarding HDL-C, TC/HDL-C, and LDL-C/HDL-C (23). LDL-C/ HDL-C ratio is an important predictor of CAD. A 1-unit change in this ratio has been reported to correspond to a $37 \%$ change in the risk of CAD. Kelishadi et al. also showed higher levels of TC, TG, and LDL-C and lower levels of HDL-C in children of parents with premature CAD (20).

In the current study, lipid parameters were analyzed among the cases and controls according to 4 age groups (0 - 5, $6-10,11-15$, and $>15$ years). The results showed a difference among all the age groups in the cases and controls regarding all the lipid parameters. The mean levels of TC and TG showed an increasing trend with age, except for $>15$ years age group, in both cases and controls. However, the mean HDL-C and TG levels were not altered significantly with age. There is increasing evidence that inflammation plays an important role in pathogenesis of atherosclerosis and its complications. CRP is a member of pentraxine family of proteins and is an acute phase reactant. There is also evidence that CRP is an independent risk factor for atherosclerosis. It is likely that hs-CRP directly participates in atherogenesis by binding to the LDL particles in atherosclerotic plaques, leading to activation of the complement system.

The findings of the present study demonstrated a significant increase in hs-CRP levels in children of patients with premature CAD. hs-CRP level was $1.2 \pm 0.77 \mathrm{mg} / \mathrm{L}$ in the cases and $0.60 \pm 0.18 \mathrm{mg} / \mathrm{L}$ in the controls, and the difference was statistically significant. Sharma SB et al. also disclosed that hs-CRP levels significantly increased in young CAD patients compared to the normal subjects (24). Moreover, Cao JJ et al. showed that addition of CRP to conventional risk factors modestly increased the ability to predict CAD (25). Guran O et al. also demonstrated that children with risk factors (hypercholesterolemia, hypertension, obesity, low HDL-C, and familial history of Coronary Heart Disease (CHD)) had significantly higher serum levels of hs-CRP compared to the control group $(3.33 \pm 4.58$ vs. $0.92 \pm 1.90 \mathrm{mg} / \mathrm{L}, \mathrm{P}<0.01)$. Thus, they concluded that serum hs-CRP level was a useful marker in screening the children at risk of CHD in adulthood (26).

The results of our study showed significantly higher hsCRP levels in the male cases compared to the female ones. The hs-CRP level was $1.58 \pm 0.84 \mathrm{mg} / \mathrm{L}$ in males and 1.03 


\section{$\pm 0.63 \mathrm{mg} / \mathrm{L}$ in females $(\mathrm{P}=0.02)$.}

The current study also assessed the relationship between carotid IMT and vascular reactivity in patients with suspected CAD and/or symptomatic hypertension. The results showed a significant difference between the cases and controls regarding IMT. However, no significant difference was found between the male and female controls concerning IMT ( $\mathrm{P}=0.93)$. Comparison of age wise distribution also revealed no significant difference in IMT levels. Furthermore, the study results indicated a significant correlation between IMT and CRP and other lipid parameters (correlation coefficient of IMT and CRP = 0.810, $\mathrm{P}<0.001$ ). Barra $\mathrm{S}$ et al. demonstrated that compared to the controls, the subjects with Parental History of Premature Myocardial Infarction (PHPMI) had increased IMT of common carotid arteries (mean of combined sites: $0.444(0.076) \mathrm{mm}$ in the cases vs. $0.382(0.062) \mathrm{mm}$ in the controls, $\mathrm{P}=0.001$ ) (27). Additionally, Yang XZ et al. showed that the incidence of increased carotid artery IMT was significantly higher $(\mathrm{P}<0.05)$ in the children with compared to those without positive family history of CAD (28). Cuomo S et al. also found that the subjects with parental history of premature MI had increased IMT of common carotid arteries (mean at $5-18$ years of age: $0.45 \pm$ $0.076 \mathrm{~mm}$ vs. $0.40 \pm 0.066 \mathrm{~mm}$ in the controls, $\mathrm{P}=0.008)(29)$. Pourafkari et al. studied 400 subjects and obtained reference values of IMT in patients without any cardiovascular risk factors (30). Moreover, Cao JJ et al. found a significant association between CRP and IMT in predicting future CAD (25). Our study also revealed a significant positive correlation among early markers of atherosclerosis, namely hs-CRP, IMT, and lipid profile $(\mathrm{P}<0.001)$.

Current studies suggest that the weight of heritability decreases whereas the influence of environmental and lifestyle factors increases with ageing (13-15). Thus, very young children of patients with early-onset atherosclerotic disease may represent an ideal study population for evaluation of the potential genes implicated in the pathogenesis of atherosclerosis. Therefore, all children of premature CAD patients, independent of sex and age groups, should be screened for dyslipidemia, so that dietary measures can be instituted as the first step towards a healthy lifestyle.

The present study had many limitations, the first of which being its small sample size. Secondly, we included the children of patients referring to a particular hospital, which might have caused selection bias. Thirdly, the cases were selected only from symptomatic patients with premature CAD, while there are asymptomatic CAD patients, as well. Thus, this may be considered a pilot study based on which, larger studies may be performed across the population to bring out a statistically powerful answer to these questions.

\subsection{Conclusions}

This study revealed that the children of patients with premature CAD had significantly higher incidence of dyslipidemia and significantly increased levels of the inflammation marker; i.e., hs-CRP, compared to the ageand sex-matched controls. This indicates that atherosclerosis is not only a disease of lipid deposition, but also an inflammatory process. This was further corroborated by demonstration of a significant rise in IMT $(\mathrm{P}<0.001)$ in the cases compared to the controls. Yet, more studies with larger sample sizes are required to be performed across the population in order to validate the findings.

\section{Acknowledgements}

This study was supported in part by a grant from Delhi University.

\section{Authors' Contribution}

Study concept and design: Gupta SK, Bindal UD; Acquisition of data: Bindal UD, Gupta SK, Daga MK, Pradhan G; Analysis and interpretation of data: Bindal UD, Bindal V; Drafting of the manuscript: Bindal UD, Bindal V; Critical revision of the manuscript for important intellectual content: Bindal UD; Statistical analysis: Bindal UD; Administrative, technical, and material support: Gupta Sk, Bindal UD; Study supervision: Gupta Sk

\section{Financial disclosure}

The funding organization is a public institution and had no role in the design and conduct of the study, collection, management, and analysis of the data, or preparation, review, and approval of the manuscript.

\section{Funding/Support}

It was supported by Delhi University.

\section{References}

1. Achari V, Thakur AK. Association of major modifiable risk factors among patients with coronary artery disease--a retrospective analysis. J Assoc Physicians India. 2004;52:103-8.

2. Mohan V, Deepa R, Rani SS, Premalatha G. Prevalence of coronary artery disease and its relationship to lipids in a selected population in South India: The Chennai Urban Population Study (CUPS No. 5). J Am Coll Cardiol. 2001;38(3):682-7.

3. Rissam H, Kishore S, Trehan N. Coronary artery disease in young Indians - the missing link. J Indian Acad Clin Med. 2001;2(3):128-31.

4. Hughes LO, Raval U, Raftery EB. First myocardial infarctions in Asian and white men. BMJ. 1989;298(6684):1345-50.

5. Enas EA, Senthilkumar A. Coronary artery disease in Asian Indians: an update and review. Coron Artery Dis. 2005;3:21-57.

6. Hamsten A, Walldius G, Dahlen G, Johansson B, De Faire U. Serum lipoproteins and apolipoproteins in young male survivors of myocardial infarction. Atherosclerosis. 1986;59(2):223-35.

7. Nikkila EA, Aro A. Family study of serum lipids and lipoproteins in coronary heart-disease. Lancet. 1973;1(7810):954-9.

8. Isser HS, Puri VK, Narain VS, Saran RK, Dwivedi SK, Singh S. Lipoprotein (a) and lipid levels in young patients with myocardial infarction and their first-degree relatives. Indian Heart $J$. 2001;53(4):463-6.

9. Pay S, Özcan N, Tokgözoğlu SL. Elevated Lp (a) is the most frequent familial lipoprotein disorder leading to premature myocardial infarction in a country with low cholesterol levels. International journal of cardiology. 1997;60(3):301-5.

10. Slyper A, Schectman G. Coronary artery disease risk factors from a genetic and developmental perspective. Arch Intern Med. 1994;154(6):633-8

11. Enas EA. Lipoprotein(a) as a determinant of coronary heart disease in young women: a stronger risk factor than diabetes? Circulation. 1998;97(3):293-5.

12. Enas EA, Dhawan J, Petkar S. Coronary artery disease in Asian Indians: Lessons learned so far and the role of Lp(a). Ind Heart J. 1997;49:25-34.

13. Sloop GD. Atherosclerosis--an inflammatory disease. $N$ Engl $J$ Med. 1999;340(24):1928.

14. Napoli C, D'Armiento FP, Mancini FP, Postiglione A, Witztum 
JL, Palumbo G, et al. Fatty streak formation occurs in human fetal aortas and is greatly enhanced by maternal hypercholesterolemia. Intimal accumulation of low density lipoprotein and its oxidation precede monocyte recruitment into early atherosclerotic lesions. $J$ Clin Invest. 1997;100(11):2680-90.

15. Rifai N, Ridker PM. High-sensitivity C-reactive protein: a novel and promising marker of coronary heart disease. Clin Chem. 2001;47(3):403-11.

16. Grundy SM, Pasternak R, Greenland P, Smith S, Jr., Fuster V. Assessment of cardiovascular risk by use of multiple-risk-factor assessment equations: a statement for healthcare professionals from the American Heart Association and the American College of Cardiology. Circulation. 1999;100(13):1481-92.

17. Ikari Y, McManus BM, Kenyon J, Schwartz SM. Neonatal intima formation in the human coronary artery. Arterioscler Thromb Vasc Biol. 1999;19(9):2036-40.

18. Strong JP, McGill HC, Jr. The pediatric aspects of atherosclerosis. J Atheroscler Res. 1969;9(3):251-65.

19. Sniderman A, Teng B, Genest J, Cianflone K, Wacholder S, Kwiterovich P, Jr. Familial aggregation and early expression of hyperapobetalipoproteinemia. Am J Cardiol. 1985;55(4):291-5.

20. Kelishadi R, Zadegan NS, Naderi GA, Asgary S, Bashardoust N. Atherosclerosis risk factors in children and adolescents with or without family history of premature coronary artery disease. Med Sci Monit. 2002;8(6):CR425-9.

21. Gulati S, Saxena A. Study of lipid profile in children of patients with premature coronary artery disease. Indian Pediatr. 2003;40(6):556-60.

22. Khalil A, Kumar D, Venkatesan M. Platelet aggregation and lipid profile in offsprings of young ischemics. Indian Pediatr.
1997;34(1):16-9.

23. Widhalm K, Koch S, Pakosta R, Schurz M, Brendinger M. Serum lipids, lipoproteins and apolipoproteins in children with and without familial history of premature coronary heart disease. $\mathrm{J} \mathrm{Am} \mathrm{Coll}$ Nutr. 1992;11 Suppl:32S-5S.

24. Sharma SB, Garg S, Veerwal A, Dwivedi S. hs-CRP and oxidative stress in young CAD patients: A pilot study. Indian J Clin Biochem. 2008;23(4):334-6.

25. Cao JJ, Arnold AM, Manolio TA, Polak JF, Psaty BM, Hirsch CH, et al. Association of carotid artery intima-media thickness, plaques, and C-reactive protein with future cardiovascular disease and allcause mortality: the Cardiovascular Health Study. Circulation. 2007;116(1):32-8.

26. Guran O, Akalin F, Ayabakan C, Dereli FY, Haklar G. Highsensitivity C-reactive protein in children at risk for coronary artery disease. Acta Paediatr. 2007;96(8):1214-9.

27. Barra S, Gaeta G, Cuomo S, Guarini P, Foglia MC, Capozzi G, et $a l$. Early increase of carotid intima-media thickness in children with parental history of premature myocardial infarction. Heart. 2009;95(8):642-5.

28. Yang XZ, Liu Y, Mi J, Tang CS, Du JB. Pre-clinical atherosclerosis evaluated by carotid artery intima-media thickness and the risk factors in children. Chin Med J (Engl). 2007;120(5):359-62.

29. Cuomo S, Guarini P, Gaeta G, De Michele M, Boeri F, Dorn J, et al. Increased carotid intima-media thickness in children-adolescents, and young adults with a parental history of premature myocardial infarction. Eur Heart J. 2002;23(17):1345-50.

30. Pourafkari M, TAMIZ BE, Jalali A, SHAKIBA M. Ultrasonic Measurement of Carotid Intima-Media Thickness in a Group of Iranian with No Cardiovascular Risk Factors. 2006. 